\title{
Androgen Receptor Signaling and the Emergence of Lethal Neuroendocrine Prostate Cancer With the Treatment-Induced Suppression of the Androgen Receptor: A Literature Review
}

Meera Dhavale ${ }^{1}$, Mohamed K Abdela ${ }^{1}$, A B M Nasibul Alam ${ }^{1}$, Tatjana Blazin ${ }^{1}$, Linha M. Mohammed ${ }^{2}$, Dhruvil Prajapati $^{1}$, Natalia P. Ballestas ${ }^{1}$, Jihan A. Mostafa ${ }^{3}$

1. Research, California Institute of Behavioral Neurosciences \& Psychology, Fairfield, USA 2. Internal Medicine, California Institute of Behavioral Neurosciences \& Psychology, Fairfield, USA 3. Psychiatry, California Institute of Behavioral Neurosciences \& Psychology, Fairfield, USA

Corresponding author: Meera Dhavale, meera94@gmail.com

\begin{abstract}
Androgen receptor signaling primarily influences both the normal growth and proliferation of the prostate gland and the development of prostatic carcinoma. While localized prostate cancers are typically managed with definitive therapies like surgery and radiotherapy, many patients have recurrences in the form of metastatic disease. Androgen deprivation therapy, by way of castration via orchiectomy or with drugs like luteinizing hormone-releasing hormone (commonly called gonadotropin-releasing hormone) agonists and luteinizing hormone-releasing hormone antagonists, is the primary mode of therapy for advanced castration-sensitive prostate cancer. Castration resistance invariably develops in these patients. Further treatment has shifted to newer anti-androgen drugs like enzalutamide or abiraterone and taxane-based chemotherapy. Prolonged inhibition of the androgen receptor signaling pathway causes androgen receptorindependent clonal evolution which leads to the development of treatment-emergent neuroendocrine prostate cancer.
\end{abstract}

All prostate cancers at the initial presentation should undergo evaluation for the markers of neuroendocrine differentiation. Detection of serum biomarkers of neuroendocrine differentiation and circulating tumor cells is a prospective non-invasive method of detecting neuroendocrine transdifferentiation in patients undergoing treatment with androgen receptor pathway inhibitors. It is essential to perform a biopsy in the presence of red flags of neuroendocrine differentiation. Alisertib, an Aurora kinase inhibitor, showed promising clinical benefit in a subgroup of patients with certain molecular alterations. A thorough understanding of the molecular and clinical programming of treatment-emergent neuroendocrine prostate cancer can potentially lead to the development of drugs to prevent the development of this lethal variant of prostate cancer.

Received 09/30/2020

Review began 02/01/2021 Review ended 02/09/2021 Published 02/17/2021

๑) Copyright 2021

Dhavale et al. This is an open access article distributed under the terms of the Creative Commons Attribution License CC-BY 4.0., which permits unrestricted use, distribution, and reproduction in any medium, provided the original author and source are credited.
Categories: Internal Medicine, Urology, Oncology

Keywords: advanced prostate cancer, neuroendocrine carcinoma of prostate, androgen receptor, castration resistant prostate cancer, androgen deprivation therapy, prostate cancer, anti-androgen therapy, treatment emergent neuroendocrine prostate cancer, neuroendocrine differentiation, aurora kinase inhibitor

\section{Introduction And Background}

Prostate cancer is the most common cancer aside from skin cancers and the second leading cause of cancerrelated death in men in the United States. About 191,930 new cases and 33,330 prostate cancer-related deaths were expected to occur in 2020 [1]. It typically begins as prostatic intraepithelial neoplasia (PIN), which transforms into localized prostate cancer. The localized prostate cancer may then transform into locally invasive advanced adenocarcinoma, leading up to metastatic prostate cancer. The aggressiveness of prostate cancer is best defined by the Gleason grading system, which grades the tumors based on histological patterns of prostatic adenocarcinoma [2].

Localized prostate cancers are primarily treated with definitive therapies, like surgery and radiotherapy. Despite the effectiveness of these therapies, $30 \%$ of patients have recurrences in the form of metastatic disease, with the five-year survival being only $29 \%$ in such cases [3].

Ever since Huggins and Hodges first demonstrated the efficacy of the technique to treat metastatic prostate cancers in 1941, androgen deprivation therapy (ADT) in the form of castration via orchiectomy or using luteinizing hormone-releasing hormone agonists (LHRH agonists) and luteinizing hormone-releasing hormone antagonists (LHRH antagonists) has been the first line of management for advanced prostate cancers [4]. ADT is also sometimes used as a neoadjuvant/adjuvant therapy with radiation [4]. The goal of androgen deprivation is to reach castration levels of testosterone $(<50 \mathrm{ng} / \mathrm{dL} ;<1.7 \mathrm{nmol} / \mathrm{L})$, which is associated with improved cause-specific survival [5]. 


\section{Cureus}

Despite primary treatment with ADT, some patients experience recurrences. These castration-resistant prostate cancers (CRPC) are usually correlated with rising prostate-specific antigen (PSA) levels, which is indicative of androgen receptor-driven activity [6]. At the moment, newer anti-androgen drugs like enzalutamide and abiraterone, and/or taxane-based chemotherapy [7,8], are used to manage CRPCs.

Substantial evidence now supports the correlation between the development of CRPC and continued transactivation of the androgen receptor [6,9]. Common mechanisms of castration resistance include alterations in the androgen receptor-signaling pathway, androgen receptor-signaling bypass mechanisms, and androgen receptor-independent clonal evolution. The latter mechanism is thought to cause the lethal form of CRPC called treatment-emergent neuroendocrine prostate cancer (t-NEPC) [10]. $t$-NEPC incidence rates are increasing with the widespread use of potent androgen receptor pathway inhibitors [6]. Table 1 summarizes the results of five research studies that chronicle the occurrences of neuroendocrine prostate cancer in patients who have undergone some form of androgen deprivation therapy.

\begin{tabular}{|c|c|c|c|}
\hline Author & Study design & $\begin{array}{l}\text { Study } \\
\text { subjects }\end{array}$ & Results \\
\hline $\begin{array}{l}\text { Beltran } \mathrm{H} \\
\text { et al. }[11]\end{array}$ & Case series & $\begin{array}{l}\text { Three } \\
\text { patients }\end{array}$ & $\begin{array}{l}\text { All patients who had previously been treated with ADT presented with low serum PSA } \\
\text { levels. Their clinical pictures corresponded to the transformation of prostatic } \\
\text { adenocarcinoma to t-NEPC. }\end{array}$ \\
\hline $\begin{array}{l}\text { Ito } \mathrm{T} \text {, et al. } \\
{[12]}\end{array}$ & $\begin{array}{l}\text { Retrospective } \\
\text { study }\end{array}$ & $\begin{array}{l}137 \text { whole } \\
\text { prostate } \\
\text { specimens }\end{array}$ & $\begin{array}{l}\text { NED markers were detected in } 70.5 \% \text { of patients who underwent ADT for }>13 \text { months } \\
\text { compared to } 30 \% \text { in those that hadn't received any ADT. }\end{array}$ \\
\hline $\begin{array}{l}\text { Aggarwal } \\
\text { R, et al. } \\
{[13]}\end{array}$ & $\begin{array}{l}\text { Multi- } \\
\text { institutional } \\
\text { prospective } \\
\text { study }\end{array}$ & 202 patients & $\begin{array}{l}\text { The study concluded that } t-N E P C \text { develops in almost one out of } 5 \text { individuals }(\sim 17 \%) \\
\text { with CRPC previously treated with ADT. }\end{array}$ \\
\hline $\begin{array}{l}\text { Hirano D, } \\
\text { et al. [14] }\end{array}$ & $\begin{array}{l}\text { Retrospective } \\
\text { study }\end{array}$ & $\begin{array}{l}93 \text { prostate } \\
\text { cancer } \\
\text { specimens }\end{array}$ & $\begin{array}{l}49 / 93(53 \%) \text { tumors tested positive for CGA. The NED status seemed to rise with longe } \\
\text { durations of treatment with ADT ( } p<0.0001) \text {. }\end{array}$ \\
\hline $\begin{array}{l}\text { Conteduca } \\
\text { V, et al. } \\
{[15]}\end{array}$ & $\begin{array}{l}\text { Prospective } \\
\text { study }\end{array}$ & specimens & $\begin{array}{l}47(54 \%) \text { NEPC cases presented de novo, \& } 40(46 \%) \text { were t-NEPC. The median time } \\
\text { from adenocarcinoma to t-NEPC diagnosis was } 39.7 \text { months with an average of } 2 \\
\text { antecedent systemic therapies. }\end{array}$ \\
\hline
\end{tabular}

TABLE 1: A summary of research articles that chronicle the occurrences of treatment-emergent neuroendocrine prostate cancer.

ADT: Androgen Deprivation Therapy; PSA: Prostate-Specific Antigen; NEPC: Neuroendocrine Prostate Cancer; t-NEPC: treatment-emergent NEPC; NED: Neuroendocrine Differentiation; CRPC: Castration-Resistant Prostate Cancer; CGA: Chromogranin A.

A delay in diagnosis and lack of specific therapies make t-NEPC a lethal form of prostate cancer with a mean survival of seven months [16]. A thorough understanding of the role of androgen receptor signaling is important as it plays a critical part in the normal growth and development of the prostate gland as well as in prostate carcinogenesis and its progression into castration-resistant cancer and neuroendocrine prostate cancer [9]. In this article, we review the physiology of androgen receptor signaling, studies that present different mechanisms of neuroendocrine transformation of prostate cancer, and potential neuroendocrine prostate cancer (NEPC)-specific markers for targeted management.

\section{Review}

\section{Androgen receptor and its signaling pathway}

Androgens like testosterone and dihydrotestosterone (DHT) are indispensable for the development of the male reproductive system and secondary sexual characteristics [17]. Androgens in adult males are primarily produced by the testes. The production of these gonadal androgens is influenced centrally by the hypothalamus and the anterior pituitary. Pulsatile secretion of LHRH from the hypothalamus drives the expression of luteinizing hormone (LH) from the anterior pituitary, which in turn stimulates the interstitial Leydig cells in the testes to produce testosterone [18]. Figure 1 describes the central regulation of androgen production. 


\section{Cureus}

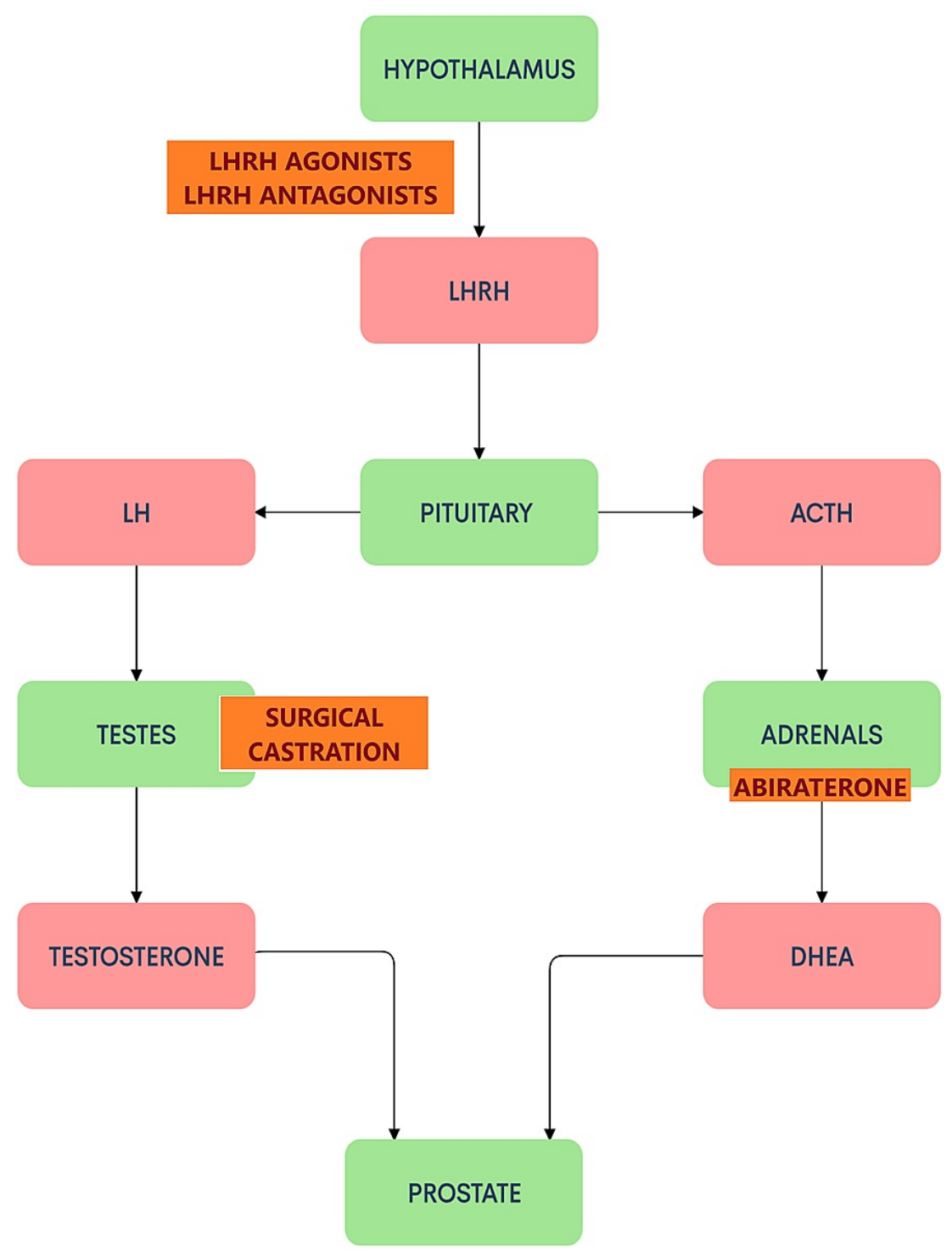

FIGURE 1: Central regulation of androgen production and the sites of action of ADT and abiraterone.

Androgen Deprivation Therapy (ADT) comprises castration by way of surgery (orchiectomy) or using drugs like LHRH agonists and LHRH antagonists [4]. Abiraterone is an antiandrogen drug that inhibits the production of androgens [19]. Both these therapies are commonly used in the treatment of prostate cancers. LHRH: Luteinizing hormone-releasing hormone; LH: Luteinizing hormone; ACTH: Adrenocorticotropic hormone; DHEA: Dehydroepiandrosterone.

Testosterone and dihydrotestosterone are steroidal hormones that exert their effects through a liganddependent nuclear transcription factor present in the cytoplasm, called the androgen receptor (AR). The enzyme 5-alpha-reductase converts testosterone to dihydrotestosterone (DHT), which is a more potent agonist of the androgen receptor [20].

The AR has three functional regions: the ligand-binding domain, the DNA binding domain, and the Nterminal domain. Endogenous androgens (like DHT) activate the receptor by attaching to its ligand-binding domain in the cytoplasm. This is followed by a conformational change in the receptor which undergoes hyperphosphorylation along with the release of heat shock proteins. The activated androgen receptor then translocates to the nucleus where the AR-ligand complex undergoes dimerization, engages with other coactivators, and undergoes DNA binding. The androgen receptor has zinc fingers that act as hormonerecognition structures for specific androgen response elements (ARE). This enables direct tethering to the promoter region of target genes [17]. Figure 2 illustrates the androgen receptor signaling and indicates the sites of action of some androgen receptor pathway inhibitors. 


\section{Cureus}

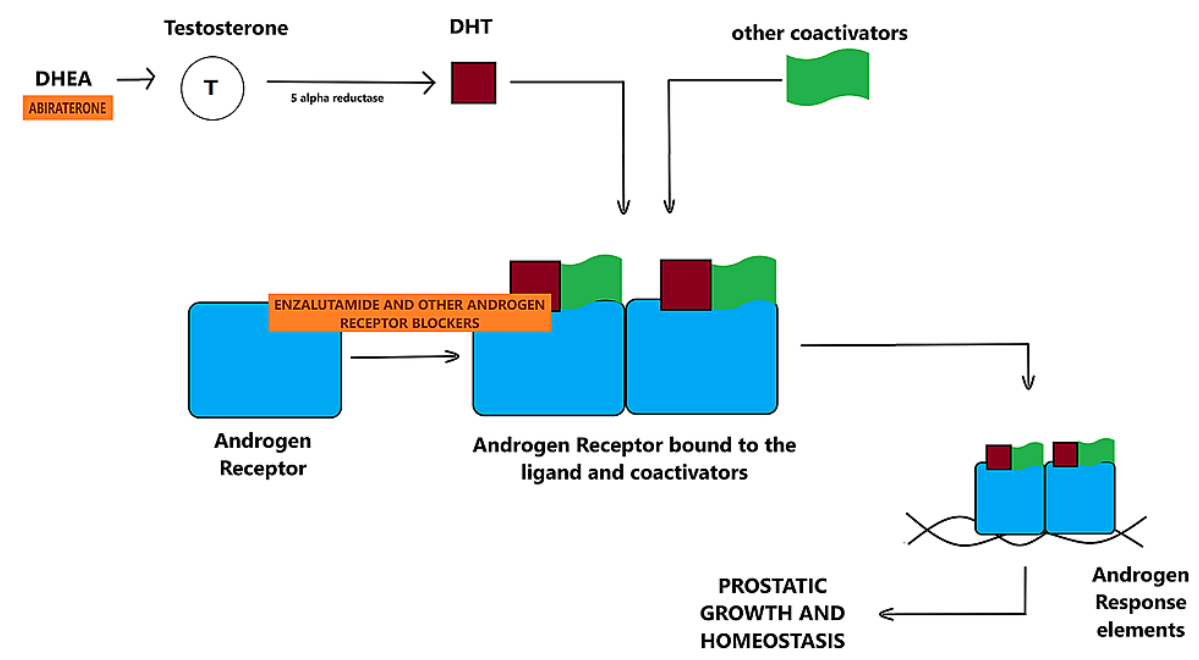

\section{FIGURE 2: Androgen receptor signaling and sites of action of abiraterone and enzalutamide.}

Enzalutamide is an androgen receptor blocker used conjointly with androgen deprivation therapy in the treatment of prostate cancers [19].

DHEA: Dehydroepiandrosterone; DHT: Dihydrotestosterone.

When a patient undergoes androgen deprivation in the form of either medical or surgical castration, the production of gonadal androgens ceases. The adrenal glands continue secreting weaker androgens like dehydroepiandrosterone (DHEA) and androstenedione. These weaker androgens supply precursors that are converted to more potent forms of androgens, like testosterone and DHT in the periphery, which go on to activate the androgen receptor. This is thought to be a mechanism of the development of CRPC [21,22].

Most patients with CRPC continue to show evidence of androgen receptor-driven activity indicated by progressive cancer with a rising PSA level [6]. Hence studies recommend combining ADT with other classes of drugs in the initial treatment of advanced castration-sensitive prostate cancers (CSPC). For this reason, newer anti-androgen drugs like abiraterone (inhibits the synthesis of testosterone) and apalutamide/enzalutamide (androgen receptor antagonists), or docetaxel (taxane-based chemotherapy), are used conjointly with ADT [22].

\section{Neuroendocrine prostate cancer}

While newer and more potent anti-androgen medications like enzalutamide and abiraterone are correlated with longer survival in patients with CRPC, resistance invariably develops to these therapies as well $[7,8]$. This occurs most commonly through alterations in the androgen receptor-signaling pathway, androgen receptor-signaling bypass mechanisms, or androgen receptor-independent clonal evolution.

Androgen receptor-independent clonal evolution gives rise to treatment-emergent neuroendocrine prostate cancer (t-NEPC) [6]. The median length of survival among patients with t-NEPC is seven months [16]. De novo small cell carcinoma of the prostate is extremely rare and resembles small cell cancers of the lung. In comparison, t-NEPC can have a pure small cell morphology or a mixed morphology since they evolve from prostatic adenocarcinoma as a mechanism of resistance $[6,23]$.

Clinical Presentation of Neuroendocrine Prostate Cancers

Aparicio et al. [24] empirically described the following seven clinical features as characteristic of neuroendocrine prostate cancer based on their experience and evidence presented by previous work [25-30]: progression to androgen-independent tumors in a short interval after androgen deprivation therapy; bulky high-grade tumor mass in the prostate or pelvis or bulky lymph nodes; histopathological confirmation of small-cell prostate cancer; low PSA at initial presentation of castration naive prostate cancer or after ADT, with abundant osseous metastases; positive staining of neuroendocrine markers like chromogranin A or synaptophysin on histological examination or elevated levels of chromogranin A in the blood in addition to the unwarranted elevation of calcium, LDH (lactate dehydrogenase), or CEA (carcinoembryonic antigen) levels in the serum; only visceral metastases; and predominant lytic osseous metastases on X-Ray or CAT scan. 
usually present with a combination of aggressive metastatic disease and low serum PSA levels. A biopsy and histopathological review are necessary to definitively diagnose NEPC and its subtypes. These cancers present with aggressive metastatic disease and biopsies of metastatic lesions are generally avoided unless neuroendocrine differentiation is suspected. The absence of markers like PSA and AR, in combination with the presence of markers of neuroendocrine differentiation (NED) like chromogranin A (CGA), neuronspecific enolase (NSE), and synaptophysin (SYP), aids the diagnosis of neuroendocrine prostate cancer $[11,31]$. A delay in diagnosis and limited treatment options make neuroendocrine prostate cancer a lethal subtype of castration-resistant prostate cancer [6].

Mechanisms of Neuroendocrine Differentiation

This paper presents a review of prior work on neuroendocrine differentiation. Following is a brief account of their aims and results:

Molecular model of neuroendocrine prostate cancer progression: Chen et al. argue that cooperation between multiple presumptive molecular drivers and facilitators is implicated in the development and progression of neuroendocrine prostate cancer [6]. According to the model presented in the paper, genes like N-Myc, RB1, and TP53 aid evasion from androgen deprivation therapy in an androgen receptor-independent manner through lineage plasticity; genes like SRRM4, REST, BRN2, and FOXA1/2 play a crucial role in specific neuroendocrine differentiation of the tumor; and mediators like AURKA, PEG10, MEAF6, and Cyclin D1 allow neuroendocrine prostate cancer cells to undergo clonal expansion and develop treatment resistance.

Cancer cell fusion and androgen-independent progression: Yin et al. studied the interaction between prostate cancer cells and neural cells using three-dimensional co-cultures of neurospheres and androgensensitive human prostatic adenocarcinoma cells. Upon induced neural differentiation, the results indicated the fusion of cancer and neural stem cells, which survived longer in a latent state. Clones of these hybrid lineages showed heterogeneity, had lost their prostatic and epithelial markers, and some had gained neural markers suggesting cancer cell and neural cell fusion in the tumor microenvironment. Random samples of these hybrid lineages showed no or subdued sensitivity to androgen stimulation, suggesting androgen receptor-independent clonal evolution [32].

Role of AURKA and MYCN (genes that code for Aurora kinase A and n-Myc respectively): Beltran et al. profiled tissue samples from neuroendocrine prostate cancer (NEPC), prostatic adenocarcinoma, and benign prostatic tissues, through ribonucleic acid (RNA) sequencing and oligonucleotide arrays using immunochemistry and fluorescence in situ hybridization (FISH). AURKA and MYCN were overexpressed in over a third of NEPC signifying they cooperate to induce neuroendocrine differentiation. Both genes were also overexpressed in local prostate cancer tissue samples, possibly indicating an at-risk group. NEPC cells responded noticeably to the administration of Aurora kinase inhibitor therapy both in vivo and in vitro, followed by the complete suppression of neuroendocrine markers. The results of the study support the potential role of Aurora kinase inhibitors in the treatment of neuroendocrine prostate cancers with these genetic alterations [33].

Role of transcription factor-4: A study conducted by Lee et al. tried to determine the mechanism of resistance of castrate-resistant prostate cancer to enzalutamide. RNA sequence analysis and examination of tissues obtained from men who had died from metastatic prostate cancer showed an association between increased levels of transcription factor-4 (TCF-4) and increased levels of neuroendocrine markers in the prostatic adenocarcinoma cell line resistant to enzalutamide when compared to the parental cell line. The results of the study stipulated that TCF-4 influences enzalutamide resistance through neuroendocrine differentiation [34].

Role of neurotensin and its receptors: A potential strategy to avoid the onset of neuroendocrine differentiation was suggested by Zhu et al. Their study argued that androgen deprivation generates neurotensin in cell lines and animal models of castrate-resistant prostate cancers with neuroendocrine features. They found that neurotensin causes neuroendocrine differentiation by the activation of neurotensin receptor 1 (NTSR1) and neurotensin receptor 3 (NTSR3), not neurotensin receptor 2 (NTSR2). Their findings indicated that inhibition of the NTSR1 signaling pathway prevented the development of neuroendocrine differentiation and castration resistance in vivo, suggesting a potential preventive strategy that can avert the onset of neuroendocrine differentiation [35].

Beta-adrenergic signaling: Sang et al. hypothesized that G protein-coupled receptor kinase 3 (GRK3) induces neuroendocrine differentiation of prostate cancer. Beta-adrenergic signaling activation causes cyclic adenosine monophosphate (CAMP) response element-binding (CREB) protein pathway activation which has been implicated in promoting angiogenesis and prostate cancer progression. Their study demonstrated that CREB activation caused an increased expression of the GRK3 and neuroendocrine markers in prostate cancers previously managed with ADT. Treatment with isoproterenol (a beta-adrenergic agonist) stimulated this pathway and propranolol (a beta-adrenergic blocker) averted the NED [36].

Leukemia inhibitory factor (LIF): The study conducted by Liu et al. argues that LIF is a formidable serum 
biomarker of advanced prostate cancer. This study demonstrated that activation of LIF signal transduction and STAT3 signaling promotes neuroendocrine differentiation of prostate cancer treated with androgen deprivation therapy. This mechanism is arbitrated by castration-induced ZBTB46 (a transcription factor that is pivotal for prostate cancer metastasis) and inhibiting the ZBTB46-LIF pathway could potentially inhibit the development of castration resistance and neuroendocrine differentiation after medical or surgical castration [37].

\section{Management of neuroendocrine prostate cancer}

Workup

While histopathological evaluation is performed at the initial presentation of prostate cancer, patients don't typically undergo evaluation for neuroendocrine prostate cancer. Newer studies recommend performing a workup for neuroendocrine prostate cancer at the initial presentation itself, by looking for neuroendocrine markers and molecular alterations implicated in the process of neuroendocrine differentiation of prostate cancers. Such a workup would rule out de novo neuroendocrine prostate cancer and aid in identifying highrisk groups [38]. Nevertheless, these recommendations are based on preclinical evidence and further research is warranted.

When NEPC presents with visceral and/or osseous metastases, biopsies of these metastatic lesions are avoided due to the belief that the risks related to the invasive procedures outweigh the benefits. However, a histopathological assessment of these metastatic tissue samples would facilitate early diagnoses. Ineffective treatments targeting the androgen receptor signaling pathway can be avoided, thereby eliminating the chance for adverse effects from the drugs and reducing treatment costs. An early diagnosis would also allow timely administration of efficacious chemotherapy to curb disease progression.

A non-invasive way of detecting t-NEPC is to identify serum biomarkers of NED. Markers like neuronspecific enolase (NSE), chromogranin A (CGA), and synaptophysin (SYP) are already being used in the histological examination of neuroendocrine prostate cancer [38]. They can be easily detected in the serum of patients with prostate cancer using techniques like enzyme-linked immunosorbent assay (ELISA). There are reports that provide evidence that CGA and NSE are upregulated in patients who have undergone hormonal therapy [39], and higher levels before or during abiraterone treatment are predictors of poorer survival $[40,41]$. Other non-invasive markers to detect neuroendocrine prostate cancer include circulating neuroendocrine tumor cells in the serum. Beltran et al. demonstrated that the circulating tumor cells from patients with neuroendocrine prostate cancer exhibited a unique structure, had subdued androgen receptor expression, and lower epithelial markers compared to those from patients with castration-resistant prostate cancer [42]. While further research is needed to corroborate these findings, these are promising results.

Treatment

There hasn't been any recent advancement in the guidelines for the treatment of neuroendocrine prostate cancer despite numerous research studies being conducted. The current recommendations advise treating them similar to anaplastic castrate-resistant prostate cancer as they have analogous clinical features. The first line of treatment for the management of localized or metastatic advanced prostate cancers is platinumbased chemotherapy which is a combination of cisplatin/carboplatin with etoposide or docetaxel [24]. While there is a high response rate to these chemotherapy regimens, they are associated with notable toxicities and don't cause long-term remission [43].

Pre-clinical studies and clinical trials are being conducted for targeted therapies for t-NEPC. A Phase II clinical trial of alisertib, an AURKA inhibitor, demonstrated significant clinical benefit in a subset of patients with AURKA and N-Myc activation [44], despite not meeting its primary endpoint. Programmed death-ligand 1 (PD-L1) inhibitors (avelumab) and Delta-like ligand 3 (DLL3) inhibitors (rovalpituzumab) are other drugs currently being studied for the treatment of NEPC [45,46]. Both PD-L1 and DLL3 inhibitors have proven useful in the treatment of small-cell lung cancers, which resemble NEPCs [47].

Clermont et al. [38] have proposed the following set of clinical guidelines that coalesces the clinical and molecular features of neuroendocrine prostate cancer. They suggest that the initial histopathological examination should also include markers of neuroendocrine differentiation (like CGA, NSE, and SYP) to rule out de novo NEPC. Advancements in research to detect sensitive molecular alterations at this stage could help predict treatment-emergent NEPC. The periodic monitoring of serum markers (like chromogranin A, neuron-specific enolase), circulating tumor cells, and circulating tumor DNA/RNA could aid in detecting the development of transdifferentiation or treatment resistance. However, further research is needed to determine their sensitivity and specificities to design better non-invasive tests. Biopsies should be promptly performed in the presence of red flags of NED like rapidly progressive lesions with disproportionate PSA levels, visceral metastases, paraneoplastic syndromes, or positive blood work findings mentioned above. As per the current standard treatment, the first line of management on confirmation of t-NEPC should be cisplatin-etoposide chemotherapy. The use of Aurora kinase A inhibitor alisertib as targeted therapy is supported by multiple clinical studies and has the potential to be included in the standard management of 
NEPC in the near future.

\section{Limitations}

As a traditional review (not a systematic review), this article didn't follow any specific protocol for data collection, and the papers included weren't subjected to quality assessment. These limitations can be a cause of researcher bias. Many studies reviewed in this article presented pre-clinical evidence, and therefore these claims presented require further investigation.

\section{Conclusions}

The adoption of newer and more potent anti-androgen medications like enzalutamide and abiraterone is expected to result in an increase in t-NEPC incidence. The diverse presentation of NEPC causes a delay in diagnosis, while limited treatment options make it lethal. Since a histopathological examination can definitively diagnose the presence of neuroendocrine differentiation, we suggest that all prostate cancers undergo evaluation for markers of NED at the initial presentation. Pre-clinical evidence suggests periodic monitoring of serum biomarkers and circulating tumor cells could aid in detecting transdifferentiation of prostatic adenocarcinoma into NEPC. It is imperative to perform a biopsy in the presence of red flags of NED.

This review paper recapitulates the role of androgen receptor signaling in the development of NEPC. It is important for physicians to look out for NED in patients with prostate cancer, especially in those who have undergone $\mathrm{ADT}$, in order to start specific therapy early in the disease progression. Apart from taxane-based chemotherapy, alisertib, an AURKA inhibitor, has demonstrated the potential to be a targeted therapy in patients with certain molecular alterations. More clinical trials and research studies need to be conducted for the development of non-invasive diagnostic tests and other targeted therapies. A thorough understanding of the molecular and clinical programming of t-NEPC can potentially lead to the development of drugs that prevent the development of this lethal variant of prostate cancer.

\section{Additional Information}

\section{Disclosures}

Conflicts of interest: In compliance with the ICMJE uniform disclosure form, all authors declare the following: Payment/services info: All authors have declared that no financial support was received from any organization for the submitted work. Financial relationships: All authors have declared that they have no financial relationships at present or within the previous three years with any organizations that might have an interest in the submitted work. Other relationships: All authors have declared that there are no other relationships or activities that could appear to have influenced the submitted work.

\section{References}

1. Cancer of the Prostate - Cancer Stat Facts . (2020). Accessed: August 12, 2020 : https://seer.cancer.gov/statfacts/html/prost.html.

2. Wang G, Zhao D, Spring DJ, DePinho RA: Genetics and biology of prostate cancer. Genes Dev. 2018, 32:1105-40. 10.1101/gad.315739.118

3. Siegel RL, Miller KD, Jemal A: Cancer statistics. CA Cancer J Clin. 2017, 67:7-30. 10.3322/caac.21387

4. Mohler JL, Antonarakis ES, Armstrong AJ, et al.: Prostate cancer, version 2.2019, NCCN clinical practice guidelines in oncology. J Natl Compr Canc Netw. 2019, 17:479-505. 10.6004/jnccn.2019.0023

5. Klotz L, O'Callaghan C, Ding K, et al.: Nadir testosterone within first year of androgen-deprivation therapy (ADT) predicts for time to castration-resistant progression: a secondary analysis of the PR-7 trial of intermittent versus continuous ADT. J Clin Oncol. 2015, 33:1151-6. 10.1200/JCO.2014.58.2973

6. Chen R, Dong X, Gleave M: Molecular model for neuroendocrine prostate cancer progression . BJU Int. 2018, 122:560-70. 10.1111/bju.14207

7. de Bono JS, Logothetis CJ, Molina A, et al.: Abiraterone and increased survival in metastatic prostate cancer . N Engl J Med. 2011, 364:1995-2005. 10.1056/NEJMoa1014618

8. Scher HI, Fizazi K, Saad F, et al.: Increased survival with enzalutamide in prostate cancer after chemotherapy. N Engl J Med. 2012, 367:1187-97. 10.1056/NEJMoa1207506

9. Lonergan PE, Tindall DJ: Androgen receptor signaling in prostate cancer development and progression . J Carcinog. 2011, 10:20. 10.4103/1477-3163.83937

10. Beltran H, Prandi D, Mosquera JM, et al.: Divergent clonal evolution of castration-resistant neuroendocrine prostate cancer. Nat Med. 2016, 22:298-305. 10.1038/nm.4045

11. Beltran H, Tagawa ST, Park K, et al.: Challenges in recognizing treatment-related neuroendocrine prostate cancer. J Clin Oncol. 2012, 30:386-9. 10.1200/TCO.2011.41.5166

12. Ito T, Yamamoto S, Ohno Y, et al.: Up-regulation of neuroendocrine differentiation in prostate cancer after androgen deprivation therapy, degree and androgen independence. Oncol Rep. 2001, 8:1221-4. 10.3892/or.8.6.1221

13. Aggarwal R, Huang J, Alumkal JJ, et al.: Clinical and genomic characterization of treatment-emergent smallcell neuroendocrine prostate cancer: a multi-institutional prospective study. J Clin Oncol. 2018, 36:2492503. 10.1200/JCO.2017.77.6880

14. Hirano D, Okada Y, Minei S, Takimoto Y, Nemoto N: Neuroendocrine differentiation in hormone refractory prostate cancer following androgen deprivation therapy. Eur Urol. 2004, 45:586-92. 
10.1016/j.eururo.2003.11.032

15. Conteduca V, Oromendia C, Eng KW, et al.: Clinical features of neuroendocrine prostate cancer . Eur J Cancer. 2019, 121:7-18. 10.1016/j.ejca.2019.08.011

16. Wang HT, Yao YH, Li BG, Tang Y, Chang JW, Zhang J: Neuroendocrine prostate cancer (NEPC) progressing from conventional prostatic adenocarcinoma: factors associated with time to development of NEPC and survival from NEPC diagnosis-a systematic review and pooled analysis. J Clin Oncol. 2014, 32:3383-90. 10.1200/JCO.2013.54.3553

17. Gottlieb B, Lombroso R, Beitel LK, Trifiro MA: Molecular pathology of the androgen receptor in male (in)fertility. Reprod Biomed Online. 2005, 10:42-8. 10.1016/s1472-6483(10)60802-4

18. Corradi PF, Corradi RB, Greene LW: Physiology of the hypothalamic pituitary gonadal axis in the male . Urol Clin North Am. 2016, 43:151-62. 10.1016/j.ucl.2016.01.001

19. Elshan NGRD, Rettig MB, Jung ME: Molecules targeting the androgen receptor (AR) signaling axis beyond the AR-Ligand binding domain. Med Res Rev. 2019, 39:910-60. 10.1002/med.21548

20. Suzuki K, Nishiyama T, Hara N, Yamana K, Takahashi K, Labrie F: Importance of the intracrine metabolism of adrenal androgens in androgen-dependent prostate cancer. Prostate Cancer Prostatic Dis. 2007, 10:301-6. 10.1038/sj.pcan. 4500956

21. Titus MA, Schell MJ, Lih FB, Tomer KB, Mohler JL: Testosterone and dihydrotestosterone tissue levels in recurrent prostate cancer. Clin Cancer Res. 2005, 11:4653-7. 10.1158/1078-0432.CCR-05-0525

22. Morris MJ, Rumble RB, Basch E, et al.: Optimizing anticancer therapy in metastatic non-castrate prostate cancer: American Society of Clinical Oncology clinical practice guideline. J Clin Oncol. 2018, 36:1521-39. 10.1200/JCO.2018.78.0619

23. Epstein JI, Amin MB, Beltran H, et al.: Proposed morphologic classification of prostate cancer with neuroendocrine differentiation. Am J Surg Pathol. 2014, 38:756-67. 10.1097/PAS.0000000000000208

24. Aparicio AM, Harzstark AL, Corn PG, et al.: Platinum-based chemotherapy for variant castrate-resistant prostate cancer. Clin Cancer Res. 2013, 19:3621-30. 10.1158/1078-0432.CCR-12-3791

25. Mahadevia PS, Ramaswamy A, Greenwald ES, Wollner DI, Markham D: Hypercalcemia in prostatic carcinoma: report of eight cases. Arch Intern Med. 1983, 143:1339-42.

10.1001/archinte.1983.00350070055010

26. Hindson DA, Knight LL, Ocker JM: Small-cell carcinoma of prostate. Transient complete remission with chemotherapy. Urology. 1985, 26:182-4. 10.1016/0090-4295(85)90060-3

27. Têtu B, Ro JY, Ayala AG, Johnson DE, Logothetis CJ, Ordonez NG: Small cell carcinoma of the prostate. Part I. A clinicopathologic study of 20 cases. Cancer. 1987, 59:1803-9. 10.1002/1097-

0142(19870515)59:10<1803::aid-cncr2820591019>3.0.c0;2-x

28. Oesterling JE, Hauzeur CG, Farrow GM: Small cell anaplastic carcinoma of the prostate: a clinical, pathological and immunohistological study of 27 patients. J Urol. 1992, 147:804-7. 10.1016/s00225347(17)37390-1

29. Amato RJ, Logothetis CJ, Hallinan R, Ro JY, Sella A, Dexeus FH: Chemotherapy for small cell carcinoma of prostatic origin. J Urol. 1992, 147:935-7. 10.1016/s0022-5347(17)37427-X

30. Papandreou CN, Daliani DD, Thall PF, et al.: Results of a phase II study with doxorubicin, etoposide, and cisplatin in patients with fully characterized small-cell carcinoma of the prostate. J Clin Oncol. 2002, 20:3072-80. 10.1200/JCO.2002.12.065

31. Huang J, Yao JL, di Sant'Agnese PA, Yang Q, Bourne PA, Na Y: Immunohistochemical characterization of neuroendocrine cells in prostate cancer. Prostate. 2006, 66:1399-406. 10.1002/pros.20434

32. Yin L, Hu P, Shi X, et al.: Cancer cell's neuroendocrine feature can be acquired through cell-cell fusion during cancer-neural stem cell interaction. Sci Rep. 2020, 10:1216. 10.1038/s41598-020-58118-z

33. Beltran H, Rickman DS, Park K, et al.: Molecular characterization of neuroendocrine prostate cancer and identification of new drug targets. Cancer Discov. 2011, 1:487-95. 10.1158/2159-8290.CD-11-0130

34. Lee GT, Rosenfeld JA, Kim WT, et al.: TCF4 induces enzalutamide resistance via neuroendocrine differentiation in prostate cancer. PLoS One. 2019, 14:0213488. 10.1371/journal.pone.0213488

35. Zhu S, Tian H, Niu X, et al.: Neurotensin and its receptors mediate neuroendocrine transdifferentiation in prostate cancer. Oncogene. 2019, 38:4875-84. 10.1038/s41388-019-0750-5

36. Sang M, Hulsurkar M, Zhang X, et al.: GRK3 is a direct target of CREB activation and regulates neuroendocrine differentiation of prostate cancer cells. Oncotarget. 2016, 7:45171-85.

10.18632/oncotarget.9359

37. Liu Y-N, Niu S, Chen W-Y, et al.: Leukemia inhibitory factor promotes castration-resistant prostate cancer and neuroendocrine differentiation by activated ZBTB46. Clin Cancer Res. 2019, 25:4128-40. 10.1158/10780432.CCR-18-3239

38. Clermont P-L, Ci X, Pandha H, Wang Y, Crea F: Treatment-emergent neuroendocrine prostate cancer: molecularly driven clinical guidelines. Int J Endocr Oncol. 2019, 6:20. 10.2217/ije-2019-0008

39. von Hardenberg J, Schwartz M, Werner T, et al.: Influence of abiraterone acetate on circulating neuromediators in chemotherapy-naïve castration-resistant prostate cancer. Prostate. 2016, 76:613-9. 10.1002/pros.23152

40. Fan L, Wang Y, Chi C, et al.: Chromogranin A and neurone-specific enolase variations during the first 3 months of abiraterone therapy predict outcomes in patients with metastatic castration-resistant prostate cancer. BJU Int. 2017, 120:226-32. 10.1111/bju.13781

41. Dong B, Fan L, Wang Y, et al.: Influence of abiraterone acetate on neuroendocrine differentiation in chemotherapy-naive metastatic castration-resistant prostate cancer. Prostate. 2017, 77:1373-80. 10.1002/pros.23397

42. Beltran H, Jendrisak A, Landers M, et al.: The initial detection and partial characterization of circulating tumor cells in neuroendocrine prostate cancer. Clin Cancer Res. 2016, 22:1510-9. 10.1158/1078-0432.CCR15-0137

43. Nadal R, Schweizer M, Kryvenko ON, Epstein JI, Eisenberger MA.: Small cell carcinoma of the prostate . Nat Rev Urol. 2014, 11:213-219. 10.1038/nrurol.2014.21

44. Beltran H, Oromendia C, Danila DC, et al.: A phase II trial of the Aurora kinase A inhibitor alisertib for 


\section{Cureus}

patients with castration-resistant and neuroendocrine prostate cancer: efficacy and biomarkers. Clin Cancer Res. 2019, 25:43-51. 10.1158/1078-0432.CCR-18-1912

45. Mansfield AS, Hong DS, Hann CL, et al.: A phase I/II study of rovalpituzumab tesirine in delta-like 3expressing, advanced solid tumors. J Clin Oncol. 2020, 38:3552-3552. 10.1200/JCO.2020.38.15_suppl.3552

46. Graff JN, Alumkal JJ, Drake CG, et al.: Early evidence of anti-PD-1 activity in enzalutamide-resistant prostate cancer. Oncotarget. 2016, 7:52810-52817. 10.18632/oncotarget.10547

47. Rickman DS, Beltran H, Demichelis F, Rubin MA: Biology and evolution of poorly differentiated neuroendocrine tumors. Nat Med. 2017, 23:1-10. 10.1038/nm.4341 